\title{
Commentaire
}

\section{Raisons pour lesquelles les données sur l'insécurité alimentaire des ménages doivent être plus détaillées}

\author{
Valerie Tarasuk, Ph. D.; Timmie Li, B. Sc.; Andrew Mitchell, M.A.; Naomi Dachner, M. Sc.
}

Diffuser cet article sur Twitter

\section{Introduction}

L'insécurité alimentaire désigne l'accès inadéquat ou précaire à une quantité suffisante de nourriture en raison de contraintes financières. L'inclusion de questions sur l'insécurité alimentaire dans les enquêtes nationales sur la santé de la population au cours des 20 dernières années a permis de bien comprendre l'épidémiologie sociale de l'insécurité alimentaire au Canada et de faire de celle-ci un indicateur d'inégalité en matière de nutrition et un puissant déterminant social de la santé. Il est de plus en plus admis qu'il est impossible de gérer les problèmes d'insécurité alimentaire des ménages au moyen d'interventions communautaires. Des interventions politiques sont nécessaires pour s'attaquer aux conditions sous-jacentes à cette vulnérabilité. La sensibilisation au thème du poids de l'insécurité alimentaire a entraîné une augmentation de la demande de renseignements sur l'étendue de ce problème.

Il est de plus en plus commun d'inclure des statistiques sur l'insécurité alimentaire dans des rapports de situation sur la santé et le bien-être de la population à l'échelle provinciale, municipale et régionale. Ces rapports sont importants, car ils permettent aux décideurs, aux organismes de planification sociale, aux autorités en matière de santé publique et à d'autres intervenants d'évaluer l'ampleur de la défavorisation au sein de leur collectivité, de surveiller les répercussions de l'évolution des conditions sociales et économiques sur la prévalence et d'établir les priorités relatives aux interventions. Cependant, compte tenu de la qualité limitée des données publiées sur l'insécurité alimentaire des ménages, il est difficile d'utiliser les données à cette fin. Cet article vise à donner un aperçu de la surveillance de l'insécurité alimentaire des ménages au Canada, à décrire les statistiques sur l'insécurité alimentaire des ménages les plus pertinentes pour la santé de la population et à relever les lacunes majeures dans les rapports actuels sur la prévalence de l'insécurité alimentaire.

\section{La mesure de l'insécurité alimentaire au Canada}

Les premières questions sur le problème de la faim chez les enfants et sur l'insuffisance alimentaire des ménages ont fait leur apparition dans les enquêtes nationales sur la santé de la population en 1994, mais l'évaluation systématique de l'insécurité alimentaire des ménages n’a démarré qu'en 2014, avec l'ajout au cycle 2.2 de l'Enquête sur la santé dans les collectivités canadiennes $\left(E C^{1}\right)$ du Module d'enquête sur la sécurité alimentaire des ménages (MESAM), une échelle de gravité à 18 points normalisée et validée élaborée par le département de l'Agriculture des États-Unis. Le MESAM a été conçu pour caractériser le niveau d'insécurité alimentaire du ménage en général. Les questions visent à connaître des expériences d'insécurité alimentaire, qui vont des préoccupations concernant le fait de manquer de nourriture avant d'avoir suffisamment d'argent pour en acheter davantage à l'incapacité financière à assurer un régime alimentaire équilibré, en passant par le fait de souffrir de la faim, de sauter des repas, et, dans les cas extrêmes, de ne pas manger pendant toute une journée en raison d'un manque de nourriture ou d'un manque d'argent pour en acheter. Le MESAM comporte 10 questions portant sur les conditions du ménage en général et sur les expériences des adultes

\section{Points saillants}

- Parallèlement à l'augmentation de la sensibilisation au poids de l'insécurité alimentaire des ménages sur la santé de la population et à sa relation aux décisions des politique publiques, les statistiques sur l'insécurité alimentaire suscitent beaucoup d’intérêt à l'échelle provinciale, municipale et régionale.

- Les données sur la prévalence de l'insécurité alimentaire auxquelles le public a accès sont insuffisantes pour répondre à l'intérêt des intervenants et leurs besoins en matière de renseignements à jour et pertinents à l'échelle locale.

- Plusieurs administrations ne mesurent pas l'insécurité alimentaire lorsque l'inclusion de ce sujet dans l'Enquête sur la santé dans les collectivités canadiennes est facultative.

- Nous recommandons de rendre obligatoire annuellement la mesure de l'insécurité alimentaire, d'exiger la production régulière de rapports sur le nombre total de personnes vivant dans un ménage en situation d'insécurité alimentaire et d'inclure l'insécurité alimentaire marginale dans l’insécurité alimentaire.

en particulier, et pour les ménages comptant des jeunes de moins de 18 ans, il comporte 8 autres questions visant à évaluer les expériences d'insécurité alimentaire des enfants.

Santé Canada a effectué une évaluation approfondie des résultats du cycle 2.2 de l'ESCC avant de confirmer la pertinence du

Rattachement des auteurs :

Département des sciences de la nutrition, Faculté de médecine, Université de Toronto, Toronto (Ontario), Canada

Correspondance : Valerie Tarasuk, Département des sciences de la nutrition, Faculté de médecine, Université de Toronto, 150, rue College, Toronto (Ontario) M5S 3E2; tél. : 416-978-0618; courriel : valerie.tarasuk@utoronto.ca 
MESAM à assurer la surveillance de l'insécurité alimentaire au Canada ${ }^{1}$. Il a aussi élaboré un schéma de classification pour définir l'insécurité alimentaire modérée et grave $^{1}$ et a proposé de classer dans la catégorie des ménages en situation de sécurité alimentaire les ménages n'ayant aucune ou une seule réponse affirmative sur l'échelle de sécurité alimentaire des adultes ou des enfants. Depuis, un grand nombre d'études révèlent que même une seule réponse affirmative à l'une des questions du MESAM est le signe d'une grande vulnérabilité ayant des conséquences importantes sur la santé et le bien-être des individus. Ces conclusions justifient que les ménages en situation marginale d'insécurité alimentaire soient considérés comme un groupe à part et soient inclus dans les estimations de la prévalence de l'insécurité alimentaire des ménages au sein la population, mais le schéma de classification de Santé Canada n’a pas encore été révisé. Comme ce schéma définit les pratiques en matière de production de rapports de Statistique Canada et de la majorité des autorités provinciales et régionales, sa mise à jour en fonction des connaissances scientifiques les plus récentes va permettre d'améliorer l'évaluation de l'insécurité alimentaire à l'échelle nationale.

\section{Surveillance de la prévalence de l'insécurité alimentaire des ménagies}

L'insécurité alimentaire est surveillée dans les cycles annuels de l'ESCC. L'échantillon de l'enquête est conçu pour être représentatif de la population canadienne de 12 ans et plus, et exclut les personnes se trouvant dans une réserve des Premières Nations, en institution, dans les Forces armées canadiennes et dans certaines régions éloignées. L’application du poids-ménage, élaboré par Statistique Canada pour tenir compte de la conception de l'échantillonnage et des profils de non-réponse, fournit un échantillon de ménages canadiens représentatif de la population pour chaque année de l'enquête, ce qui permet d'estimer la proportion et le nombre de ménages ayant fait état d'insécurité alimentaire au cours de l'année. Cette pondération, apte à estimer la prévalence à l'échelle nationale, provinciale et territoriale, n’a pas été calibrée pour être utilisée à l'échelle infraprovinciale et infraterritoriale, ce qui limite la détermination de la prévalence de l'insécurité alimentaire à l'échelle régionale ou locale.
Le tableau 1 illustre la prévalence de l'insécurité alimentaire au Canada en 20072008 et en 2011-2012, soit les deux cycles pour lesquels il existe des données représentatives à l'échelle nationale. Que les ménages en situation marginale d'insécurité alimentaire soient considérés comme en situation d'insécurité alimentaire ou non, leur prévalence a connu une croissance marquée au cours de la période. Compte tenu du fait qu'il a été largement prouvé que l'insécurité alimentaire est néfaste pour la santé, cette augmentation devrait être prise très au sérieux.

\section{Estimation du nombre de personnes vivant dans un ménage en situation d'insécurité alimentaire}

Outre surveiller la prévalence de l'insécurité alimentaire des ménages, il est essentiel de faire le suivi du nombre de personnes exposées à l'insécurité alimentaire au sein de chaque ménage, car c'est cette exposition qui constitue un indice très important de la santé et du bien-être des individus. Le fait de surveiller le nombre ou la proportion de personnes (et non de ménages) touchées par l'insécurité alimentaire est cohérent également avec la mesure d'autres statistiques de population (p. ex. le taux de pauvreté infantile, le taux de chômage ou le nombre de personnes ayant recours aux banques d'alimentation). C'est sans doute ce qui explique que ce soit la méthode la plus employée pour décrire l'insécurité alimentaire des ménages dans les publications de base et dans les rapports de situation publiés par de nombreux organismes de planification sociale, d'autorités en matière de santé publique et d'autres groupes d'intervenants. Cependant, avoir accès à ces données est complexe, car l'échantillon de l'ESCC n'inclut que les personnes de 12 ans et plus.

Grâce à l'application du poids-personne, on peut facilement, à partir des données de l'ESCC, obtenir une estimation représentative de la population du nombre de personnes de 12 ans et plus vivant dans un

TABLEAU 1

Nombre et proportion de ménages ayant déclaré être en situation d'insécurité alimentaire et nombre de personnes qui vivaient dans un ménage en situation d'insécurité alimentaire au Canada en 2007-2008 et en 2011-2012

\begin{tabular}{lrr} 
& $2007-2008$ & $2011-2012$ \\
\hline Tous les ménages & $446000(3,6 \%)$ & $536200(4,1 \%)$ \\
\hline Insécurité alimentaire marginale & $626300(5,1 \%)$ & $759600(5,8 \%)$ \\
Insécurité alimentaire modérée & $329500(2,7 \%)$ & $333500(2,5 \%)$ \\
Insécurité alimentaire grave & $1401800(11,3 \%)^{\mathrm{a}}$ & $1629400(12,4 \%)^{\mathrm{a}}$ \\
\hline Total & 1155100 & 1389800 \\
\hline Toutes les personnes & 1570800 & 1897600 \\
\hline Insécurité alimentaire marginale & 684200 & 652000 \\
Insécurité alimentaire modérée & 3410200 & 3939500 \\
Insécurité alimentaire grave & & 1107100 \\
\hline Total & 932700 & 1518700 \\
Personnes de 12 ans et plus & 1237200 & 537300 \\
\hline Insécurité alimentaire marginale & 556300 & 3163000 \\
Insécurité alimentaire modérée & 2726300 & 282800 \\
Insécurité alimentaire grave & & 378900 \\
\hline Total & 222400 & 114800 \\
\hline Personnes de moins de 12 ans & 333500 & 776500 \\
\hline Insécurité alimentaire marginale & 127900 & \\
Insécurité alimentaire modérée & 683800 & \\
Insécurité alimentaire grave & & \\
\hline Total & & \\
\hline
\end{tabular}

Remarque : Les chiffres ayant été arrondis, leur somme peut ne pas correspondre au total indiqué.

a Un test du chi carré des proportions révèle une différence importante, $p<0,0001$. Lorsque l'insécurité alimentaire marginale est exclue de la prévalence totale de l'insécurité alimentaire, le test du chi carré de la différence entre les cycles donne un résultat important, $p=0,0017$. 
ménage en situation d'insécurité alimentaire. Cependant, cette méthode sousestime grandement le nombre total de personnes touchées et masque la vulnérabilité accrue des jeunes enfants à l'insécurité alimentaire de leur ménage. On peut estimer le nombre total de personnes vivant dans un ménage en situation d'insécurité alimentaire en appliquant le poids-ménage aux données de l'ESCC sur la composition des ménages. Grâce à cette méthode, nous avons conclu que 3939500 Canadiens vivaient dans un ménage en situation d'insécurité alimentaire en 2011-2012, soit 529300 personnes de plus qu'en 2007-2008 (tableau 1). En outre, plus d'une personne sur cinq vivant dans un ménage en situation d'insécurité alimentaire était un enfant de moins de 12 ans.

Les variables nécessaires à l'estimation de la prévalence de l'insécurité alimentaire dans les ménages et le nombre total de personnes vivant dans un ménage en situation d'insécurité alimentaire se trouvent dans les fichiers maitres de l'ESCC, auxquels ont accès les chercheurs dans les Centres de données de recherche de Statistique Canada, ainsi que dans les fichiers partagés, auxquels ont accès les gouvernements provinciaux et territoriaux, mais elles ne se trouvent pas dans les fichiers à usage public sur l'ESCC. Par conséquent, les organismes n'ayant pas la capacité ni l'autorisation d'analyser les microdonnées de l'ESCC n'ont pas accès à ces données.

\section{La fréquence de la mesure de l'insécurité alimentaire}

Le MESAM fait partie du contenu de base des cycles de l'ESCC de 2007-2008, de 20112012 et de 2017-2018 mais, pour les années entre ces cycles, la décision d'utiliser le module revient aux gouvernements provinciaux et territoriaux. Seuls l'Alberta, le Québec, la Nouvelle-Écosse, les Territoires du Nord-Ouest et le Nunavut ont choisi de mesurer l'insécurité alimentaire des ménages au cours de chaque cycle depuis l'intégration du MESAM en 2005. Les taux de prévalence de l'insécurité alimentaire au Canada ne sont pas stables, comme le révèle l'augmentation importante de la prévalence à l'échelle nationale entre 20072008 et 2011-2012 : le taux d'insécurité alimentaire varie en fonction de l'évolution des conditions macroéconomiques ${ }^{2-3}$ et des décisions politiques ayant des répercussions financières sur les ménages ${ }^{3-6}$.
Les décisions des gouvernements provinciaux et territoriaux de cesser de mesurer l'insécurité alimentaire empêchent les décideurs, les autorités en matière de santé publique et divers autres intervenants des provinces et des territoires concernés d'utiliser ces données pour évaluer l'ampleur de la défavorisation à l'intérieur de leurs frontières et d'établir des priorités fondées sur des données probantes en ce qui a trait aux programmes et aux interventions relatives aux politiques. Les lacunes dans ces mesures nuisent également aux activités de recherche et d'évaluation visant à connaître les conséquences de décisions politiques précises sur la prévalence et la gravité de l'insécurité alimentaire. Nous pouvons comprendre l'incidence des décisions politiques fédérales et provinciales sur ce problème uniquement si nous pouvons comparer les taux de prévalence avant et après chaque réforme politique $e^{3-6}$. En l'absence de données préalables et postérieures aux interventions, comment pouvons-nous mieux comprendre ce qui fonctionne? La mesure sporadique de l'insécurité alimentaire retarde le développement des connaissances nécessaires à la conception d'interventions politiques fondées sur des données probantes visant à régler un problème de santé de la population grave, très répandu et en augmentation.

\section{L'accessibilité de l'information sur la prévalence de l'insécurité alimentaire}

Les tableaux de CANSIM permettent de consulter en ligne les estimations de la prévalence à la fois de l'insécurité alimentaire des ménages modérée et grave $^{7}$ et celle des personnes de 12 ans et plus qui vivaient dans un ménage en situation d'insécurité alimentaire modérée ou grave en 2007-2008 et en 2011-2012 ${ }^{8}$. Ces estimations existent à l'échelle nationale, provinciale et territoriale, et diverses estimations sous-provinciales ont été utilisées pour le calcul de l'exposition des personnes de 12 ans et plus en fonction du poidspersonne. Santé Canada a publié des statistiques sommaires sur l'insécurité alimentaire modérée et grave dans les provinces et les territoires qui ont participé aux activités de mesure en 2005 et en 2009-2010, et a publié en août 2017 des tableaux présentant des estimations de la prévalence de l'insécurité alimentaire dans les provinces et les territoires pour 2013-2014.
Certains gouvernements provinciaux et territoriaux et certaines autorités locales de la santé ont publié des rapports sur le taux d'insécurité alimentaire à l'intérieur de leurs frontières pour les années autres que celles incluses dans CANSIM, mais ces rapports utilisent généralement les données publiées par la base de données CANSIM de Statistique Canada. À quelques rares exceptions près, les taux d'insécurité alimentaire modérée et grave sont combinés, les ménages en situation marginale d'insécurité alimentaire sont considérés comme étant en situation de sécurité alimentaire et l'exposition à l'insécurité alimentaire est fournie uniquement pour les personnes de 12 ans et plus. En outre, on ne sait pas si une administration à une échelle quelconque s'est engagée à produire régulièrement et en temps utile des rapports sur l'insécurité alimentaire.

Dans le cadre de la stratégie d'application des connaissances de notre programme de recherche financé par les Instituts de recherche en santé du Canada (IRSC), intitulé " PROOF », nous avons produit des rapports plus détaillés sur la prévalence de l'insécurité alimentaire des ménages au Canada en 2011, en 2012, en 2013 et en 2014, p. ex. ${ }^{9}$. L'immense intérêt que suscitent ces rapports laisse penser qu'ils ont comblé un vide important pour les intervenants et la population en général. Cependant, les travaux de transfert des connaissances réalisés dans le cadre d'une initiative de recherche à durée limitée ne peuvent pas remplacer la publication régulière et en temps utile de données sur l'insécurité alimentaire sur les sites Internet du gouvernement.

\section{Conclusion et recommandations}

Comme les connaissances sur les conséquences de l'insécurité alimentaires des ménages sur la santé et la société au Canada progressent et comme les liens entre ce problème et les décisions des politiques publiques sont mieux compris, surveiller l'insécurité alimentaire apparaît de plus en plus comme essentiel. Les seules mesures représentatives à l'échelle nationale dont nous disposons révèlent une augmentation marquée du nombre de Canadiens dans un ménage en situation d'insécurité alimentaire entre 2007-2008 et 2011-2012, ce qui souligne la nécessité de faire preuve dorénavant d'une vigilance accrue dans la surveillance de ce problème. 
Pour connaître l'ampleur de l'insécurité alimentaire des ménages au Canada, il faut surveiller à la fois la proportion de ménages en situation d'insécurité alimentaire et le nombre de personnes vivant dans un ménage en situation d'insécurité alimentaire. Les rapports publics sur l'exposition à l'insécurité alimentaire sont encore limités aux personnes de 12 ans et plus qui vivent dans un ménage en situation d'insécurité alimentaire modérée et grave. Aucune donnée n'est actuellement accessible sur la prévalence des ménages en situation d'insécurité alimentaire à l'échelle régionale et municipale, et les ménages en situation marginale d'insécurité alimentaire continuent à être considérés comme des ménages en situation de sécurité alimentaire. En outre, comme la mesure de l'insécurité alimentaire est facultative dans la plupart des cycles de l'ESCC, d'une part, il est impossible de s'appuyer sur ces données pour agir dans les administrations qui décident de ne pas mesurer ce problème, et d'autre part, ce caractère facultatif nuit aux recherches nécessaires à l'élaboration de solutions efficaces pour remédier à l'insécurité alimentaire à l'échelle nationale ainsi qu'à l'échelle provinciale et territoriale.

Pour combler les lacunes relatives à la collecte de données et à la production des rapports mentionnées précédemment, nous recommandons la prise de mesures suivantes :

i. faire en sorte que les statistiques sur l'insécurité alimentaire publiées régulièrement dans les tableaux de CANSIM comprennent des données sur le nombre total de personnes vivant dans un ménage en situation d'insécurité alimentaire;

ii. faire en sorte que la situation marginale d'insécurité alimentaire soit reconnue comme une situation d'insécurité alimentaire et incluse dans les calculs correspondant de prévalence;

iii. si possible, calibrer le poids-ménage pour l'ESCC à l'échelle sous-provinciale et sous-territoriale afin de rendre accessibles davantage de données locales sur l'insécurité alimentaire;

iv. faire en sorte que l'évaluation de l'insécurité alimentaire des ménages fasse partie du contenu de base de tous les cycles de l'ESCC.

\section{Remerciements}

Cette recherche a été financée grâce à une subvention programmatique pour la santé et l'équité en santé des Instituts de recherche en santé du Canada (IRSC) (FRN 115208). Les auteurs expriment leur reconnaissance à Andrée-Anne Fafard St-Germain pour ses commentaires d'une importance cruciale sur les versions antérieures de cet article.

\section{Conflits d'intérêts}

Les auteurs déclarent n'avoir aucun conflit d'intérêts.

\section{Avis}

Le contenu de cet article et les opinions qui y sont exprimées n'engagent que les auteurs et ne sont pas forcément représentatifs de la position du Gouvernement du Canada.

\section{Références}

1. Santé Canada. Enquête sur la santé dans les collectivités canadiennes, cycle 2.2, Nutrition (2004) : Sécurité alimentaire liée au revenu dans les ménages canadiens. Ottawa (Ont.) : Santé Canada; 2007. [rapport no 4696]

2. Sriram U, Tarasuk V. Changes in household food insecurity rates among Census metropolitan areas from 2007 to 2012. Can J Public Health. 2015; 106(5):e322-e327.

3. Emery J, Bartoo A, Matheson J, Ferrer A, Kirkpatrick S, Tarasuk V, et al. Evidence of the association between household food insecurity and heating cost inflation in Canada, 1998-2001. Canadian Public Policy. 2012;38(2): 181-215.

4. Loopstra R, Dachner N, Tarasuk V. An exploration of the unprecedented decline in the prevalence of household food insecurity in Newfoundland and Labrador, 2007-2012. Canadian Public Policy. 2015;41(3):191-206.

5. Li N, Dachner N, Tarasuk V. The impact of changes in social policies on household food insecurity in British Columbia, 2005-2012. Preventive Medicine. 2016;93:151-158.
6. Ionescu-Ittu R, Glymour M, Kaufman J. A difference-in-difference approach to estimate the effect of income-supplementation on food insecurity. Prev Med. 2015;70:108-116.

7. Statistique Canada. Base de données CANSIM : Tableau 105-0546 - Mesures de l'insécurité alimentaire du ménage, selon la présence d'enfants dans le ménage, Canada, provinces et territoires, occasionnel (nombre) [Internet]. Ottawa (Ont.) : Statistique Canada; 2013 [mise à jour le 11 décembre 2013; consultation le 28 avril 2017]. En ligne à : http://www5.statcan.gc.ca/cansim /a26?lang = fra\&id = 1050546\&retrLang $=$ fra

8. Statistique Canada. Base de données CANSIM : Tableau 105-0547 - Insécurité alimentaire du ménage, selon le groupe d'âge et le sexe, Canada, provinces, territoires, régions sociosanitaires (limites de 2013) et groupes de régions homologues, occasionnel (nombre) [Internet]. Ottawa (Ont.) : Statistique Canada; 2013 [mise à jour le 11 décembre 2013; consultation le 28 avril 2017]. En ligne à : http:// www5.statcan.gc.ca/cansim/a26?lang $=$ fra\&id $=1050547 \&$ retrLang $=$ fra

9. Tarasuk V, Mitchell A, Dachner N. Household food insecurity in Canada, 2012. Toronto (Ont.) : Research to Identify Policy Options to Reduce Food Insecurity (PROOF); 2014. 